\title{
IMPORTANCIA Y SENTIDO DEL COACHING Y LA INTELIGENCIA EMOCIONAL EN ITINERARIOS PARA EL EMPLEO DE PERSONAS CON DISCAPACIDAD INTELECTUAL
}

\section{IMPORTANCE AND MEANING OF COACHING AND EMOTIONAL INTELLIGENCE IN ITINERARIES FOR THE EMPLOYMENT OF PEOPLE WITH INTELLECTUAL DISABILITIES}

\author{
María Lorena Lucas Moreno. Universitat de Barcelona. España. \\ llucas@afanias.org
}

\section{RESUMEN}

Coaching e inteligencia emocional se presentan como dos potentes recursos a utilizar en los itinerarios para el empleo de personas con discapacidad intelectual. El artículo que nos ocupa ofrece, por un lado, una presentación de lo que es el coaching, pilares básicos y profesionales referentes, y cómo sus principios conectan -en el contexto de los itinerarios para el empleo de las personas con discapacidad intelectual-, con la planificación centrada en la persona, enfoque metodológico que las organizaciones del sector social vienen utilizando para la puesta en marcha del desarrollo del proyecto vital y empoderamiento personal de cada una de las personas de este colectivo. A su vez, se realiza un análisis del significado de inteligencia emocional y de la importancia de este constructo impartido en las acciones formativas de los itinerarios para el empleo, como elemento trampolín que forma y capacita para el autoconocimiento, la autorregulación, la toma de decisiones y la capacidad de relacionarse favorablemente con los otros, en todo tipo de entornos y en particular en los laborales. Con la necesidad de contextualizar lo anteriormente expuesto se lleva a cabo una presentación del concepto discapacidad intelectual y algunas de las leyes que amparan gran parte de los derechos del colectivo. Se explica qué es y en qué consiste un itinerario laboral para el empleo de personas con discapacidad intelectual, sus fases, contenidos y objetivos principales, tomando como referente el de una entidad social que trabaja en pro de la calidad de vida de las personas con discapacidad intelectual y sus familias.

PALABRAS CLAVE: Apoyo, coaching, discapacidad intelectual, inteligencia emocional, itinerarios para el empleo, planificación

\section{ABSTRACT}

Coaching and emotional intelligence are presented as two powerful resources waiting to use in the itineraries for the employment of people with intellectual disabilities. The present article offers us, on one side an introduction to the definition of coaching, basic pillars, professional referents and how their principles connect - in the context of itineraries for the hiring of people with mental impairment -, with planning focused on the individual and a methodological focus that companies in the social sector are using to deploy the development of life projects and personal empowerment for each and all of the people in this collective. Likewise, an analysis 
on the definition of emotional intelligence and the importance of this construct is carried out. That is taught in the training activities for hiring itineraries as a "jumpstart" to train and capacitate self-learning, self-regulation, decision making and the ability to establish mutually favourable relationships with others, in all types of environments, particularly in those related to work. With the need to contextualize the aforementioned, a presentation of the concept of intellectual disability and some of the laws that protect a large part of the rights of the group is carried out. And explanation is given on what is and what defines a work itinerary for people with intellectual impairment, the steps to take, contents and main objectives, taking as a referent a Social Entity from Madrid that dedicates itself to the well-being of people that are mentally impaired and their families.

KEYWORDS: Support, coaching, intellectual disability, emotional intelligence, itineraries for employment, planning.

\section{Cómo citar el artículo:}

Lucas Moreno, M. L. (2020). Importancia y sentido del coaching y la inteligencia emocional en itinerarios para el empleo de personas con discapacidad intelectual. Revista de Ciencias de la Comunicación e Información, 25(2), 41-53. doi: http://doi.org/10.35742/rcci.2020.25(2).41-53

\section{INTRODUCCION}

Todas las personas necesitamos sentirnos felices, sea cual sea nuestro color de piel, condición, género u origen. Necesitamos tras tener cubiertas las necesidades básicas, sentirnos realizadas, saber que formamos parte de la comunidad en la que vivimos y considerarnos útiles. Formar parte activa de la sociedad y ejercitar el derecho a tener un empleo y mantenerlo en el tiempo, es para muchos individuos máximo indicador de inclusión social y calidad de vida. "Debemos meditar sobre lo que proporciona la felicidad, pues, estando ella presente, lo tenemos todo, y estando ausente, lo hacemos todo para alcanzarla". (Epicuro, 1998)

Cuando se logran satisfacer las necesidades básicas, surgen en las personas nuevos deseos, nuevas necesidades más elaboradas y complejas, y así sucesivamente. (Maslow, 1987)

\subsection{Contextualizando la discapacidad intelectual}

Las personas con discapacidad intelectual no se muestran ajenas a esta fórmula universal. Acceder a un empleo y mantenerlo en el tiempo es para muchas de ellas un indicador de felicidad, un reflejo de la tan ansiada inclusión social. Por ello, la formación en itinerarios para el empleo es objetivo de un gran número de entidades u organizaciones sociales dedicadas a facilitar la calidad de vida de este colectivo.

Según la Asociación Americana de discapacidades intelectuales y del desarrollo (AAIDD, 2011) la discapacidad intelectual se caracteriza por limitaciones significativas en el funcionamiento intelectual y en la conducta adaptativa 
manifestada en las habilidades adaptativas conceptuales, sociales y prácticas. Esta discapacidad se origina antes de los 18 años.

Esta definición enfoca la discapacidad intelectual desde un enfoque ecológico, centrándose en la relación persona-ambiente, y en la mejora que la persona puede tener en función de los apoyos que recibe (Shalock et cols, 2007).

El colectivo de personas con discapacidad intelectual demanda, cada vez con mayor rotundidad su derecho igualitario a ser, estar y contribuir a la colectividad, amparados por la Ley General de Discapacidad.

La Ley General de Discapacidad (LGD), Real Decreto Legislativo 1/2013 de 29 de noviembre tiene como objetivo, entre otros, fomentar la igualdad de oportunidades en el acceso al empleo, y promover los incentivos económicos y fiscales que, entre otras razones, impulsan a la contratación de personas con discapacidad en las empresas. La contratación de personas con discapacidad, además, aporta un valor social a las empresas indiscutible.

La citada ley plantea, a su vez, que toda empresa que contrate a personas con discapacidad podrá beneficiarse de ayudas públicas para la adaptación de puestos de trabajo y/o eliminación de barreras arquitectónicas. Se establece para las empresas públicas y privadas, con una plantilla superior a 50 trabajadores, la obligación de contratar a un número de trabajadores con discapacidad no inferior al $2 \%$.

Según Plena Inclusión Madrid, en España viven aproximadamente 230.000 personas con algún tipo de discapacidad intelectual y alrededor de 50.000 son susceptibles de ser empleadas: 10.000 de ellas ya trabajan en Centros Especiales de Empleo; otras 5.000 han accedido al empleo ordinario, y las restantes 35.000 se encuentran sin empleo. En el caso de las personas con más necesidades de apoyo, estas cifras de desempleo y tasa de inactividad se disparan (Plena Inclusión, 2020).

Un adecuado ajuste del perfil del trabajador frente al perfil del puesto de trabajo generará beneficios indiscutibles tanto para la persona como para la empresa. Por ello resulta necesaria la formación en itinerarios para el empleo de personas con discapacidad intelectual, que provean a este colectivo de herramientas que posibiliten una formación sólida, coherente y ajustada a su realidad y expectativas frente a las necesidades del entorno laboral.

El modelo de itinerario para el empleo en este artículo está inspirado en el llevado a cabo por el Área de Formación y Empleo de la entidad AFANIAS, que trabaja en pro de la calidad de vida de las personas con discapacidad intelectual y sus familias.

\section{OBJETIVOS}

El presente trabajo tiene como objetivos definir el concepto de discapacidad intelectual y contextualizarlo a través de la Ley General de Discapacidad. Se pretende presentar qué son los itinerarios para el empleo de personas con discapacidad intelectual, y hacer una reflexión y análisis de los principios del 
coaching, y de cómo éstos conectan con el enfoque metodológico que hoy en día llevan a cabo las organizaciones del sector de atención a personas con discapacidad intelectual, planificación centrada en la persona, en concreto en el escenario de los itinerarios para el empleo.

Por último, se propone poner en valor el concepto de inteligencia emocional como materia necesaria y obligatoria en las acciones formativas de los itinerarios para el empleo de las personas con discapacidad, en la fase denominada "Formación en habilidades personales, sociales y prelaborales".

\section{METODOLOGÍA}

A través de la descripción y la explicación así como de algunas referencias teóricas y bibliográficas se reflexiona acerca de las posibilidades que coaching e inteligencia emocional ofrecen como herramientas posibilitadoras de cambio y empoderamiento personal.

\section{ITINERARIOS PARA EL EMPLEO}

Las empresas empleadoras demandan profesionales formados, maduros y competentes, y la educación formal no da respuesta a las necesidades formativas de las personas con discapacidad intelectual, por ello, una vez finalizada, en torno a los 21 años, se precisa de una formación adaptada y significativa que dé continuidad a su itinerario personal y profesional. (Fundación Iberoamericana Down 21.org, 2020)

Según el informe "El reto de la integración del joven con discapacidad" (2015), de la Fundación Adecco, el 62\% de jóvenes entre 18 y 30 años con alguna discapacidad está en situación de desempleo. La complejidad de la inserción sociolaboral de este colectivo justifica la necesidad de planificar un conjunto de acciones formativas centradas, entre otras, en habilidades y competencias pre-laborales.

El acceso a un puesto de trabajo requiere estar preparado y formado adecuadamente en estrategias, habilidades y competencias personales y profesionales. Por tanto, ofrecer orientación, formación y apoyo a las personas que acceden a los itinerarios de empleo es obligación de las entidades que trabajan en pro del acceso al empleo de las personas con discapacidad intelectual. La finalidad es fomentar la adquisición y generalización de competencias personales, sociales y laborales que garanticen su inclusión en la comunidad, el ejercicio de su ciudadanía y el acceso al empleo, todo ello en pro de su calidad de vida.

Los itinerarios para el empleo se dividen en diferentes etapas o fases (Formación y Empleo Afanias, 2020).

a) Acogida y Valoración de la Empleabilidad: a través de una primera entrevista y la observación se recogen aspectos relacionados con la identificación, datos familiares, formación, experiencia laboral, datos laborales, expectativas con respecto a la formación y el empleo, limitaciones funcionales, actividades de la vida diaria, habilidades sociales básicas, etc, así como la valoración de la 
empleabilidad a través de cuestionarios, por ejemplo el método de perfiles Lantegi Batuak ${ }^{1}$. Esta información garantiza el diagnóstico de empleabilidad.

b) Orientación centrada en la persona: a través de una o más entrevistas se lleva a cabo una orientación que determinará el proceso pedagógico a seguir orientado al cambio de la persona, de la situación actual a la deseada: a través de formación prelaboral, o bien formación específica en un perfil concreto, o si la persona está ya lista para empleo, la participación en sesiones de búsqueda de empleo sistemáticas.

c) Formación en Habilidades Personales, Sociales y Prelaborales: se trata de acciones formativas que permiten adquirir y desarrollar actitudes, habilidades y estrategias intra e interpersonales a través del conocimiento personal y el desarrollo de habilidades sociales a fin de favorecer la adecuada inserción laboral; así como los conocimientos y las habilidades necesarias para elegir, obtener y mantener un trabajo y propiciar la iniciativa de búsqueda de empleo, la intercomunicación y las relaciones grupales y la utilización de las herramientas necesarias para la búsqueda de empleo.

- -Habilidades Personales y Sociales: son acciones formativas orientadas a desarrollar la inteligencia emocional, en las que se trabajan la identificación de sentimientos y estados emocionales propios y ajenos, la motivación personal, la aceptación de limitaciones y el ajuste de expectativas, el autoconcepto, la empatía, el autocontrol y tolerancia a la frustración, el control emocional y la relajación, la toma de decisiones, los prejuicios, los diferentes estilos de comunicación, la comunicación verbal y no verbal, la escucha activa, los estilos de comportamiento, y la resolución de conflictos. Todo ello en pro de una conducta adaptativa e inteligente emocionalmente, en cualquier entorno y aplicable, sobre todo, a entornos laborales.

- -Habilidades Prelaborales: son acciones formativas orientadas a desarrollar la motivación para el trabajo, hábitos laborales básicos, el valor del trabajo, el conocimiento de la estructura jerárquica y las relaciones existentes, la gestión del tiempo y el entrenamiento para la búsqueda de empleo: herramientas básicas, fuentes de información laboral para la búsqueda, la entrevista personal y pruebas de selección.

d) Formación Específica para el Empleo: posibilita capacitar a las personas en competencias teórico-prácticas en perfiles laborales específicos, ajustados a las motivaciones, habilidades y aptitudes de la persona en ese instante justo de su itinerario vital, por ejemplo: formación teórico-práctica en perfil profesional auxiliar de oficina, formación teórico-práctica en perfil profesional azafato de eventos, formación teórico práctica en perfil profesional auxiliar de office, formación teórico-práctica en perfil profesional limpieza, formación teórico-práctica en perfil profesional cajero-reponedor, formación teóricopráctica en perfil profesional digitalizador de datos, etc.

e) Búsqueda Activa de Empleo: se trata de sesiones sistemáticas grupales o individuales de acceso a portales de empleo especializados en ofertas para personas con certificado de discapacidad: previo registro se hace una búsqueda de las posiciones más acordes con el perfil profesional de cada

\footnotetext{
${ }^{1}$ Lantegi Batuak es una organización no lucrativa que genera oportunidades laborales adaptadas a las personas con discapacidad, preferentemente intelectual.
} 
participante. Cada participante envía su currículum actualizado a la empresa ofertante.

El proceso de los itinerarios para el empleo es facilitado por un profesional referente desde la fase primera a la última, el orientador, que se muestra como profesional conductor y conector de unas fases con otras. Es el profesional que guía las reuniones y fomenta la participación de todos los miembros, personas y profesionales, mediante un diálogo respetuoso y una actitud positiva, creativa y productiva.

\subsection{Coaching}

Etimológicamente coaching tiene su origen en la ciudad de Kocs, en el siglo XV, cuando esta ciudad era parada estratégica y obligada en el trayecto Viena-Budapest. El carruaje que trasladaba de una ciudad a la otra se denominaba kocsi, en español "coche". Simbólicamente, en el proceso de coaching se traslada a la persona (cliente) de un estado $X$ inicial, hacia un estado deseado.

Se trata de un proceso de acompañamiento a personas, equipos u organizaciones, de carácter no directivo, que posibilita la exploración y gestión de los cambios que desean llevar a cabo con el fin de alcanzar los objetivos que se propongan.

Según Gallwey (2013), consiste en "liberar el potencial de las personas, para que puedan llevar su rendimiento al máximo."

Es un proceso que genera transformación en las personas o clientes a través de la conversación y de preguntas poderosamente efectivas que fomentan la toma de conciencia. La mayéutica socrática es uno de sus primeros antecedentes. Las preguntas permiten analizar dónde está la persona y dónde quiere llegar, poniendo en marcha un plan de acción, que sigue cuatro etapas diferenciadas:

a) Establecimiento del objetivo de la sesión. b) Comprobación de la realidad para estudiar la situación. c) Valoración de las opciones y las estrategias o actuaciones alternativas. d) Determinar qué se va a hacer, cuándo se va a hacer y quién lo realizará.

El coaching, como indica Whitmore $(2017$, p.19) "se centra en las posibilidades del futuro, no en los errores del pasado".

Las preguntas que se llevan a cabo a la persona se hacen en positivo. El profesional o coach se centra en lo que sí quiere o puede hacer el cliente. ej: ¿qué deseas obtener de esta sesión? ¿Qué te gustaría lograr en este tiempo?

Se trata de interrogaciones que llevan inherentes un sentido, relacionadas con hacia dónde quiere ir la persona, ej: ¿Qué es lo que quieres concretamente? ¿Qué es exactamente lo que verás, oirás o sentirás cuando lo alcances? ¿Qué es lo que se alcanzará realmente? ¿En qué contexto? ¿Cuándo quieres alcanzarlo? ¿Con quién? ¿Dónde? ¿Cómo lo quieres conseguir? 
Teniendo muy presente que cada persona es única e irrepetible, con su propio mapa mental fruto de las experiencias personales vitales diferentes y únicas. "Así como toda persona tiene un conjunto distinto de huellas dactilares, cada individuo tiene también experiencias nuevas al crecer y vivir, y ninguna historia de vida es idéntica a otra". (Bandler y Grinder, p. 32)

Se tratan de preguntas medibles y comparables, que orientan hacia el conocimiento de un resultado: ¿cómo vas a medir que lo has conseguido? ¿Cómo sabrás que estás en el buen camino? Si se alcanza el resultado, ¿a qué se parece? ¿Qué te demuestra que hay un resultado? ¿Qué pasa si no lo hay?

Preguntas con un enfoque ajustado a la realidad, ¿Hasta qué punto controlas ese objetivo? ¿En qué medida depende de ti? ¿Cuál es el compromiso? ¿Con qué recursos cuentas? ¿Qué recursos te faltan? ¿Cómo los vas a conseguir? ¿Cuáles son los pasos? ¿Qué etapas vas seguir?

Que se orientan hacia un momento en el tiempo, ubican en la temporalidad, ej: ¿Cuándo quieres alcanzarlo? ¿Cuál es la fecha de inicio? ¿Cuáles son las etapas intermediarias a corto plazo?

Desde una perspectiva ecológica, ¿Cuáles son las consecuencias para otras personas? ¿A qué podrías tener que renunciar? ¿Qué cosas importantes de tu vida actual tal vez tengas que dejar atrás?

Y visibilizan la recompensa, ej, ¿Qué es lo que te importa a ti en este objetivo? ¿Qué obtienes haciendo esto? ¿Quién serás cuando lo consigas?

Según Whitmore, (2017, p.19) "los resultados que ofrece el coaching se deben, en gran medida, a la relación de apoyo que se establece entre el coach y el cliente, y al estilo y los medios de comunicación que se emplean". Destaca, según el mismo autor, la capacidad del profesional de creer en el potencial del otro, en lo mejor del otro y de todas sus posibilidades.

La visión que el profesional adopta del otro y sus potencialidades es optimista, y pone el foco en las fortalezas de la persona. Cuanto más auténtico que se muestre el profesional en la relación más útil resulta (Rogers, 1987).

Se trata, por tanto, como indica este autor de un proceso en el que destaca la no directividad, a través del cual la naturaleza del ser humano se manifiesta libremente, en confianza y de forma constructiva. El cliente, ha de asumir la responsabilidad de tomar decisiones libremente, no bajo el mandato del profesional. Él mismo va creando su propio itinerario.

La importancia de que la persona tome conciencia, "se dé cuenta", es otro aspecto fundamental del coaching, el cliente ha de llegar a ser capaz de identificar los cambios que quiere implementar en su vida, y para eso es preciso el autoconocimiento, saber qué se dice a sí mismo y cómo, conocer sus creencias y cuáles son los obstáculos que le impiden llegar a conseguir lo que desea (creencias limitantes). 
El obstáculo interno es invariablemente universal y único, y se describe como el miedo al fracaso, la falta de autoconfianza, seguridad o autoestima se convierten en obstáculos universales. Hay que dejar claro al cliente que delante de ellos hay profesionales, personas, que creen en ellos, los apoyan y acompañan para que tomen sus decisiones (Whitmore, 2017).

El coaching posibilita la capacidad de conocerse a uno mismo, de autoconocimiento, y gracias a ello podemos actuar de manera más eficiente y relacionarnos mejor con nosotros mismos y con los demás (Fraile, 2013). Solo tomando conciencia de lo que nos sucede podemos llegar a controlarlo.

\subsection{Planificación centrada en la persona}

Planificación centrada en la persona es la metodología que impera en los servicios de atención a personas con discapacidad intelectual, donde el enfoque está puesto en la individualidad de la personas como tal, y en la capacidad y derecho de éstas a escoger sus futuros planes vitales así como a personas significativas para que les brinden el apoyo para llevarlos a cabo. Todo ello basado en el modelo de calidad de vida de Shalock y Verdugo (2003).

Según Plena Inclusión, la Planificación Centrada en la Persona continúa siendo el principal enfoque en el apoyo a personas con diversas discapacidades, al ofrecer estrategias basadas en el empoderamiento y valor de éstas, así como a facilitadores que acompañan en la construcción de su propio proyecto de vida plena, de felicidad. El punto de partida de este enfoque es el reconocimiento de la dignidad de la persona, más allá de las condiciones vitales de cada una.

Las estrategias y los medios para llevar a cabo esta metodología van a depender exclusivamente de cada caso. Las entidades responsables adoptan el compromiso de proveer a las personas con discapacidad intelectual de las herramientas y apoyos que posibiliten proyectos de vida encaminados a la felicidad de las personas.

El proyecto vital de cada una vendrá determinado por lo que cada cual entienda por felicidad, definido esto, a su vez, por sus creencias, valores, aspiraciones y contexto. La felicidad no puede ser impuesta desde el exterior ni definida igual para todos, guarda relación con lo que el propio individuo entiende por lo que es una vida plena (Shalock y Verdugo, 2003).

Citando a Álex Rovira (2005, p.97), "la felicidad es esa sensación de que mi vida tiene una dirección y un sentido, de que está guiada por una brújula interior". El objetivo de la planificación centrada en la persona es lograr una vida de calidad en la que las decisiones propias sean las que determinen el itinerario vital.

Según Shalock y Verdugo (2003) la calidad de vida es multidimensional y está influida por factores personales, del entorno y sus interacciones, presenta los mismos componentes en todas las personas, y son tanto subjetivos como objetivos. Mejora con la autodeterminación, la disposición de recursos, metas vitales y el sentimiento de pertenencia. Sus dimensiones e indicadores son: 
- Bienestar emocional: satisfacción, autoconcepto, falta de estrés.

- Relaciones interpersonales: Interacciones, relaciones, apoyos.

- Bienestar material: situación económica, empleo, alojamiento.

- Desarrollo personal: educación, competencia personal, rendimiento.

- Bienestar físico: cuidados, estado de salud, actividad cotidiana, entretenimiento y ocio.

- Autodeterminación: autonomía o autocontrol, metas y valores.

- Inclusión social: integración, participación en la comunidad, apoyos sociales, roles comunitarios.

- Derechos: situación legal y humana (dignidad y respeto).

Según Plena Inclusión, "podemos definir PCP como un proceso continuo de escucha, centrado en los intereses de cada persona, en sus capacidades y en sus expectativas y sueños, que genera acciones concretas para un cambio personal y social, contando con el compromiso de los miembros del grupo de apoyo y asegurando el protagonismo y participación activa de la persona para establecer y perseguir metas personales". (Carratalá, Mata y Crespo, 2017, p.10)

\subsection{Inteligencia Emocional}

La inteligencia ha sido estudiada a lo largo de los años por un amplio número de autores, psicólogos, filósofos, etc. Hasta ahora el cociente intelectual era el que determinaba el éxito profesional de una persona, y en teoría el desempeño académico, sin embargo se fue descubriendo que el éxito en la vida venía determinado por otras cuestiones más allá de un cociente intelectual. Estas capacidades eran de difícil medición y tenían mucho que ver con el adecuado manejo de las emociones. "Conocer a los demás es sabiduría, conocerse uno mismo es iluminación". (Lao Tse, S. V a. C)

El origen de la inteligencia emocional se lo debemos a varios autores a lo largo de la historia, que de una manera u otra hicieron alusión al término abundando los modelos interpretativos diferentes. Howard Gardner será quien conocido por su famosa teoría de las inteligencias múltiples asienta el germen del constructo inteligencia emocional. Según él, es necesaria la ruptura con el concepto psicométrico de inteligencia y propone que ésta deje de estar asociada únicamente al cociente intelectual iniciando una nueva línea de investigación que reconsidere los avances realizados por las ciencias cognitivas.

Gardner (1995) propone siete inteligencias distintas que recogen la forma en que los individuos procesan, adquieren y retienen la información con el medio:

- Inteligencia lingüística, considerada una de las más importantes. La amplia utilización del lenguaje es parte fundamental para el desarrollo de este tipo de inteligencia

- la inteligencia lógico-matemática, inteligencia empleada en la resolución de problemas matemáticos, permite formular y verificar hipótesis, calcular, utilizar el método científico y el razonamiento deductivo e inductivo. 
- inteligencia musical, habilidad musical que se traduce en capacidad para cantar y tocar instrumentos.

- inteligencia espacial, es la que posibilita hacer un modelo mental en tres dimensiones del mundo. Permite la comprensión de mapas, orientarse, etc.

- inteligencia cinestésico-corporal, propia de bailarines, gimnastas, deportistas, en esta inteligencia destaca el control y la expresión corporal.

- inteligencia intrapersonal, nos permite comprendernos a nosotros mismos, formando una imagen precisa de quiénes somos, cuáles son nuestras características, nuestras fortalezas y cuáles nuestras debilidades. Nos sirve como guía de comportamiento y conducta en el devenir vital.

- inteligencia interpersonal, es el tipo de inteligencia que nos permite entender a los demás, sus motivaciones, deseos, estados de ánimo. Está fundamentada en la empatía, es decir, en la capacidad de ponerse en el lugar del otro.

Bar-On (1998) acuñará el término cociente emocional y creará el inventario emocional del cociente (EQ-i), que es la primera prueba testada de la Inteligencia Emocional en su tesis doctoral "The developmnet of a concept of psychological wellbeing". El modelo está compuesto por diversos aspectos: componente intrapersonal, componente interpersonal, componente del estado de ánimo en general, componentes de adaptabilidad, componentes del manejo del estrés y, componente del estado de ánimo en general. Su tesis constituyó la base de sus posteriores formulaciones sobre la inteligencia emocional y su medida a través del inventario emocional del cociente EQ-I, que es la primera prueba testada de la inteligencia emocional.

Mayer, Salovey y Carusso (2000) definen la inteligencia emocional como la capacidad de asimilar, percibir, comprender y regular las emociones, la habilidad de procesar la información emocional eficazmente.

Daniel Goleman (2006) populariza el término manifestando la idea de que una gestión buena, positiva de las emociones, es más determinante en la vida que el coeficiente intelectual.

Según Barrientos (2019), la inteligencia emocional radica en la capacidad de controlar y gestionar positivamente las emociones propias y ajenas, en un escenario cualquiera, donde se producen experiencias y cambios como parte del proceso de aprendizaje personal.

La inteligencia emocional nos ayuda a manejar nuestros impulsos y comprender cómo sienten los otros.

Según dicho autor, los principales elementos que integran la inteligencia emocional serían:

1. Autoconocimiento o conciencia de uno mismo: es importante conocer cuáles son nuestras fortalezas o puntos fuertes así como nuestras debilidades, y cómo las emociones y los sentimientos pueden condicionar nuestra conducta. 
2. Autocontrol o autorregulación: tomar conciencia de nuestras dinámicas emocionales y ser capaces de dominar nuestras emociones o sentimientos forma parte de esta capacidad de adaptarnos al entorno.

3. Motivación: Tener la capacidad de dar un sentido significativo a cuanto hacemos y orientar los objetivos hacia una meta, un fin ventajoso y premiante.

4. Empatía: Tener la capacidad de ponernos en el lugar del otro, capacidad de detectar los sentimientos ajenos. Detectar e interpretar de forma correcta las emociones ajenas y sus sentimientos nos ayuda a establecer vínculos más estrechos, auténticos y duraderos. Son precisamente las personas con mayores habilidades y competencias relacionadas con la inteligencia emocional.

5. Relaciones interpersonales/ Habilidades sociales: La capacidad de relacionarse con los otros adecuadamente -comunicación asertiva- garantiza éxito en cualquiera de los entornos y en cualquier tipo de relación.

Goleman destaca que la persona que es emocionalmente inteligente se muestra eficaz en todas y cada una de las áreas citadas, la persona emocionalmente inteligente precisa mostrar competencia en todas ellas, como un todo.

La inteligencia emocional se aprende y se puede potenciar.

\section{CONCLUSIONES}

Todo individuo busca ser dueño de su vida y de su felicidad. Para un gran número de personas con discapacidad intelectual en edad de trabajar la felicidad radica en tener la posibilidad de acceder al empleo y mantenerlo en el tiempo.

Los itinerarios para el empleo son procesos formativos que dan respuesta real a las necesidades de cada una de las personas con discapacidad intelectual en edad de trabajar, se trata de un conjunto de acciones formativas que, en sus diferentes fases permiten la valoración y la orientación posterior a formación específica de las personas en función de sus intereses, motivaciones, fortalezas, etc.

Coaching e inteligencia emocional se presentan como dos herramientas potentes y significativas en los itinerarios para el empleo.

El coaching porque sus principios rectores conectan de lleno con los del enfoque de la Planificación Centrada en la Persona (metodología que se viene empleando en la mayor parte de organizaciones y servicios que atienden a personas con discapacidad intelectual): ambos ofrecen estrategias que buscan el empoderamiento de los individuos, y el reconocimiento de éstos como seres únicos y excepcionales dueños de su propia vida y destino.

Ambas perspectivas se centran en las capacidades de las personas, y en las posibilidades de éstas. Atribuyen poder a su voluntad, a la libertad, y a la capacidad de elegir, y en consecuencia a la responsabilidad de tomar las decisiones que considere oportunas para poder llevar a cabo el cambio deseado. Ambas disciplinas o enfoques apuestan por la no directividad y el acompañamiento a la persona en su 
proceso de cambio. Ambas, se presentan como metodologías ecológicas, tienen en cuenta la red comunitaria, el entorno y el efecto del cambio en su red de relaciones.

Ambos enfoques buscan dar un sentido a la vida de las personas, y a través de la inteligencia emocional desarrollar la autoestima, la seguridad y la toma de consciencia de uno mismo para poder establecer objetivos a posteriori.

La inteligencia emocional, a su vez, se presenta como recurso potente en las acciones formativas de los itinerarios para el empleo, pues permite dotar a los futuros trabajadores de herramientas necesarias para el autoconocimiento, así como la capacidad de desarrollar habilidades personales, sociales e interpersonales óptimas, a través de potenciar el autoconocimiento, la empatía, la asertividad, la motivación y el autocontrol, entre otros. El aprendizaje y la puesta en práctica de estos mecanismos posibilitan el empoderamiento y la correcta adaptación de la persona a todo tipo de entornos, en particular a los de empleo.

\section{BIBLIOGRAFIA}

A handbook for human service practitioners. American Association on Mental Retardation, 2002.

AAIDD (2011). Discapacidad intelectual. Definición, clasificación y sistemas de apoyo social. Alianza Editorial.

Bandler, R. y Grinder, J. (2007). La estructura de la magia. Vol. I Lenguaje y terapia. Cuatrovientos. Chile.

Bar-On, R. (1988). The development of a concept of psychological well-being. Unpublished. doctoral dissertation, Rhodes University, South Africa.

Barrientos-Báez, A., Barquero-Cabrero, M. y Rodríguez-Terceño, J. (2019). Educación Emocional como contenido transversal para una nueva política educativa: el caso del Grado de Turismo. Revista Utopía y Praxis Latinoamericana, 24(4), 147-165. ISSN 1315-5216 / ISSN-e: 2477-9555

Carratalá, A., Mata, R. y Crespo S. (2017). Planificación Centrada en la Persona. Materiales de apoyo para facilitar procesos de PCP con personas con discapacidad intelectual y edad avanzada. Madrid. Plena Inclusión España.

Cuadri Fernández, J., Fierro Suero, S. y Palma Rodríguez, I. (2015). El coaching. La educación en valores y la práctica de actividad física en colectivos en situación de desigualdad. E-motion. Revista de Educación, Motricidad e Investigación, 4, 1833.

Epicuro (1998). Obras. Altaya.

Formación y Empleo, Afanias (2020). Etapas o fases de Itinerarios para el empleo. Material no publicado. 
Fraile Aranda, A. (2013). El coaching como estrategia pedagógica para la mejora del autoconocimiento de los educadores deportivos. E-motion. Revista de Educación, Motricidad e Investigación, 1, 1-13.

Fundación Adecco (2015). El reto de la integración del joven con discapacidad.

Fundación Iberoamericana Down 21.org. Por qué es importante que trabajen las personas con discapacidad intelectual.

Gallwey, T. (2013). El juego interior del tenis. Ed. Sirio.

Gardner, H. (1995). Inteligencias múltiples. La teoría en la práctica. Paidós.

Goleman, D. (2006). Inteligencia Emocional. Kairós.

Maslow, A. H. (1987). Motivation and Personality (3rd ed., revised by R. Frager, J. Fadiman, C. McReynolds, y R. Cox. Harper \& Row.

Mayer, J.D., Salovey, P. y Caruso, D.R. (2000). Models of emotional intelligence. In R.J. Sternberg (Ed.), Handbook of intelligence. Cambridge University Press.

Pérez, E. (2017). \#Todoal27. La formación como oportunidad de inclusión de las personas con discapacidad intelectual. Plena Inclusión.

Rogers, C. (1987). El proceso de convertirse en persona. Paidós.

Román López, M. T. (2008). Lao Tse y el Tao te Ching. Espacio, Tiempo y Forma II, Historia Antigua, 21, 39-50.

Rovira Celma, Á. (2005). La brújula interior. Conocimiento y éxito duradero. Ed. Urano.

Schalock, R. L. y Verdugo, M. A. (2003). Calidad de vida. Manual para profesionales de la educación, salud y servicios sociales. Alianza.

Whitmore, J. (2017). Coaching. El método para mejorar el rendimiento de las personas. Los principios y la práctica del coaching y del liderazgo. Paidós.

\section{AUTORA}

María Lorena Lucas Moreno

Universitat de Barcelona. España.

llucas@afanias.org

\section{Google Scholar:}

https://scholar.google.es/citations?view op=list works\&hl=es\&user=WJ8sPh0AAAAJ 